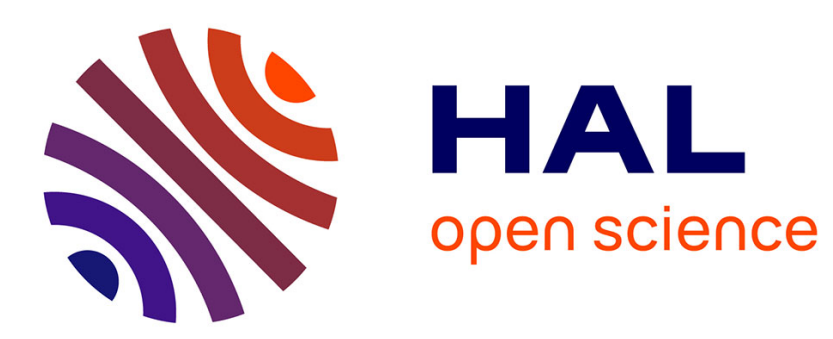

\title{
Software for Modelling Positron-Beam Depth-Profiling Data
}

\author{
H. Schut, A. van Veen
}

\section{To cite this version:}

H. Schut, A. van Veen. Software for Modelling Positron-Beam Depth-Profiling Data. Journal de Physique IV Proceedings, 1995, 05 (C1), pp.C1-57-C1-61. 10.1051/jp4:1995106 . jpa-00253542

\section{HAL Id: jpa-00253542 https://hal.science/jpa-00253542}

Submitted on 1 Jan 1995

HAL is a multi-disciplinary open access archive for the deposit and dissemination of scientific research documents, whether they are published or not. The documents may come from teaching and research institutions in France or abroad, or from public or private research centers.
L'archive ouverte pluridisciplinaire HAL, est destinée au dépôt et à la diffusion de documents scientifiques de niveau recherche, publiés ou non, émanant des établissements d'enseignement et de recherche français ou étrangers, des laboratoires publics ou privés. 


\title{
Software for Modelling Positron-Beam Depth-Profiling Data
}

\author{
H. Schut and A. van Veen
}

Interfaculty Reactor Institute, Mekelweg 15, $2629 \mathrm{JB}$ Delft, The Netherlands

\begin{abstract}
The fitting program VEPFIT has been extended with applications running under the Microsoft Windows environment facilitating the input and output of the VEPFIT fitting module. We have exploited the Microsoft-Windows ${ }^{\circledR}$ graphical users interface by making use of dialog windows, scrollbars, command buttons etc. The user communicates with the program simply by clicking and dragging with the mouse pointing device. Keyboard actions are limited to a minimum. Upon changing one or more input parameters the results of the modeling of the S-parameter and Ps-fraction versus positron implantation energy are updated and displayed. This action can be considered as the first step in the fitting procedure upon which the user can decide to further adapt the input parameters or to forward these parameters as initial values to the fitting routine. The modeling step has proven to be helpful for designing positron beam experiments.
\end{abstract}

\section{INTRODUCTION}

Positron beams with variable energy provide a sensitive tool for the investigation of the depth distribution of defects in solid materials. Applications are found in semiconductor technology where the quality of epitaxial layers and interfaces is of importance $[1,2,3]$, and in the study of irradiation induced damage in fission and fusion materials [4]. The positron beam method is usually based on the measurement of the Doppler broadening of the $511 \mathrm{keV}$ photopeak and the determination of the positronium fraction. In order to translate these experimental data into the relevant depth dependent parameters characterizing the material one has to solve the positron transport equation. This equation takes into account the implantation, diffusion, drift, and trapping of positrons. By imposing the appropriate boundary conditions surface related processes such as positron emission, positron surface trapping and positronium formation can be incorporated.

In 1990 we have introduced the VEPFIT software package [5], which is capable of solving this problem. Meanwhile, several adjustments and extentions have been added, some of which are presented at this workshop [6]. Partly based on our own experience and on comments of other VEPFIT users we have decided to improve the communication with the program and to extend it with a modeling stage. The adaptations presented here concern the building of a Windows shell around the existing MS-DOS ${ }^{\mathbb{1}}$-version of VEPFIT. In this Windows shell the 
input and output routines for the main fitting routine are processed. In addition, on request of the user the modeling cycle is executed and its results are displayed on screen. The input routine is written in Microsoft-Visual Basic ${ }^{\circledR}$ (VB) for Windows. The graphical presentation and the processing of the modeling stage are performed in the Microsoft-Excel ${ }^{\circledR}$ for Windows environment. The fitting itself is still performed under DOS.

In the next section the different parts of the software package, their use and limitations will be described. The overall performance and future developments will be addressed in section III. Finally, in section IV the hardware and software requirements for using this version of VEPFIT will be presented.

\section{PROGRAM DESCRIPTION}

The program is divided into three individual modules. The fitting module is called VEXC.EXE and runs under MS-DOS. The source is written in FORTRAN-code, which is compiled and linked with the Lahey Fortran Compiler/Linker. Apart from minor changes in the input-file format description it is identical to the earlier DOS version of VEPFIT. The input module VEPLAY is written in Visual Basic code and is therefore a full-fledged Windows application. The executable VEPLAY.EXE is created from this code and runs even without Visual Basic being present. The third module consists of several Excel documents, such as worksheets, charts and macros. The modeling is performed in a Excel worksheet. It is the Excel-equivalent of the Fortran-code, but without the routines necessary to perform fitting. Cells in this sheet contain, besides input and output data (e.g. S-parameter, positron fraction and positron energy), the matrix and vector elements resulting from the depth discretization applied to solve the transport equation (see [5]). Data in this sheet are visualized by the chart VEPMOD.XLC. This chart can also contain experimental data files. They are displayed on request by the user in module VEPLAY. Two macro documents are included. The macro START.XLM opens all relevant Excel documents and starts the input module. During the rest of the processing it remains inactive. The second macro VEPFIT.XLM takes care of starting the fit procedure, switching between windows and returning to the input module.

Data transfer between the separate parts of the program depends on the environment in which the serving and/or receiving modules are operating. Within Excel the data are transferred by remote referencing; cells in one sheet or ranges of cells in a chart adopt values from cells or ranges in another sheet. Data transfer between the Visual Basic input module and the modeling module is performed by Dynamic Data Exchange (DDE) [7]. Communication beween the two window applications and the fitting module is performed through ASCII-files by means of read and write instructions. The communication between the user and the VEPFIT package to set up the modeling (and thus the fitting parameters) goes through the input module. Upon initiating this module a screen with scrollbars, push buttons, and input fields is activated and displayed. The complete contents of this screen is shown in figure 1. Clicking (or double clicking) with the mouse on selected screen objects (the controls) results in the execution of a subroutine (procedure) which corresponds to this control. A description of the objects, events and the result of the procedures is given below. The subsequent actions performed by the user to set up the modeling will act as a guide.

The user may start by clicking and dragging on the scrollbar in the left-hand top corner. This event defines the number of layers in the model. As yet, only a layered structure can be modeled (this corresponds to model number 5 in the DOS VEPFIT version). A total of six layers can be inserted. The program responds by showing the selected number of layers and an extra layer representing the vacuum-solid interface. 


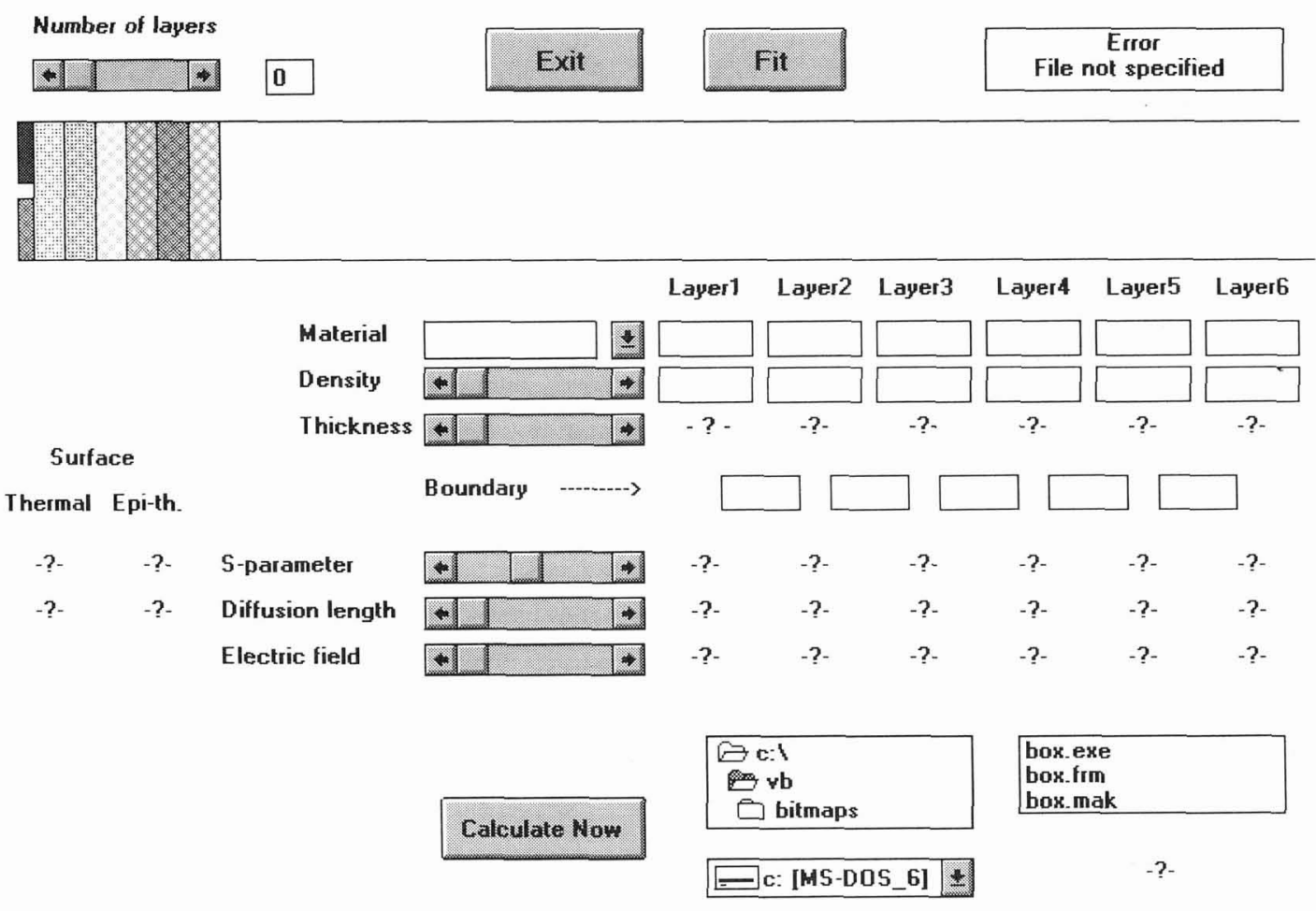

Fig.1. An overview of the setup sereen of the Visial Basic input module VEPLAY. Starting in the left hand top corner we see a scrollbar for selecting the number of layers in the model. To the right of this there are a push button to exit the program and a button to forward the parameter values to the fitting routine and start the fitting. In the right hand top corner an area for displaying error messages is shown. Below this a schematic diagram of the layered structure and a surface layer split into a thermal and an epi-thermal field are shown. The drop-down box "Material" contains elements to setup the layers using default values. These values can be changed by activating the scrollbars for density, layer thickness, S-parameter, diffusion length and electric field strength. The values are displayed in the areas containing a question mark. The "Calculate Now" button shows the result of the modeling step. This can be compared with experimental data taken from the file shown in the file name box in the right hand bottom corner.

The next event is double-clicking on one of the layers. This event activates a drop-down box showing a list of selected chemical elements, compounds or the text "interface". By choosing one of these items the text fields containing the values of the material density of the layer, the S-parameter, the diffusion length and the electric field strength are updated with (user supplied) default values. In addition, the text "layer \#", where \# is the number of the selected layer, is high-lighted. This indicates that scrollbar actions only relate to this layer. Increasing or decreasing the layer thickness is visualized by the changing width of the selected layer. If more than one layer is present, the depth position of the boundary between two adjacent layers is shown too. Once a layer has been defined the next layer can be set up, 
following the same procedure. Note that double clicking results in reverting to the default values. In order to change selected values of layer parameters one has to single click on the layer, followed by setting the corresponding scrollbars.

Changing the surface parameters for thermal or epi-thermal positrons is achieved by clicking on one of the surface areas. One can adjust the thermal and epi-thermal surface S-parameters, the epi-thermal scattering length or the surface-absorption length. The scrollbar captions are updated according to the surface area chosen.

By pushing the "Calculate now" button the parameter values are sent to the Excel sheet containing the modeling code and the result is shown as an S-parameter versus positron energy curve. One also has the option to display one or more experimental data files. This is achieved by activating the drop-down boxes showing the active disk drive, working directory and data file. Double clicking on the selected file(s) updates the graphics screen with the data and legends.

Finally, the parameter values shown (including the filename displayed in the filename box) are forwarded to the fitting routine by pushing the "FIT" button. In case one or more relevant parameters are missing this procedure is cancelled and an error message is displayed. A green background color of an area containing a parameter value indicates that this parameter can be fitted. A default white background means that the parameter is kept fixed at the indicated value. The user can toggle between the two fit options by clicking on the areas. After the fitting has been performed three buttons are shown. These allow the user to display and subsequently print the fit results (as graphics or as text) or to return to the setup screen.

\section{PROGRAM PERFORMANCE}

The modeling option of this VEPFIT-version enables the user to explore easily the effect of changes in the parameter setup which is assumed to characterize the sample under study. For example, the number of layers included in a layered structure can be extended with interfacial layers to investigate the relevance of the interfaces to the measured positron data. In case of a series of measurements on a single sample with, e.g., one experimental parameter changed, the modeling stage allows the user to determine wich parameters can be fixed to obtain a consistent set of fitting results. Here one may think of a series of S-parameter measurements on a MOS-device as a function of the applied gate-voltage.

As stated earlier the present version only enables the modeling (and fitting) of a layered structure. Future development will include the option of a defect profile, a detailed description of electric fields for semiconductor (MOS) studies and the incorporation of material dependent positron stopping profiles.

\section{REQUIREMENTS}

To be able to run this version of VEPFIT the following software and hardware is required: Software: the VEPFIT-Windows software package (including the Visiual Basic input module, VBRUNxxx and Excel files), Windows 3.0 or later, Excel 4.0 and MS-DOS 3.1 or later. Hardware: an IBM PC/AT compatible computer with VGA graphics card, a mouse pointing device, a (fast) hard disk and $4 \mathrm{MB}$ or more memory. A printer is strongly recommended. 


\section{REFERENCES}

[1] Schut H., van Veen A., van $\operatorname{der}$ Walle G.F.A. and van Gorkum A.A., J. Appl. Phys. 70 (1991) 3003.

[2] Gossmann H.J., Asoka-Kumar P, Leung T.C., Nielsen B., Lynn K.G., Unterwald F.C., and Feldman L.C., Appl. Phys. Lett. 61 (1992) 540.

[3] Schultz P.J., Tandberg E., Lynn K.G., Nielsen B., Jackman T.E., Denhoff M., and Aers G.C., Phys. Rev Lett. 61 (1988) 187.

[4] Evans H.E., Rice-Evans P., Evans J.H., Smith D.L., and Smith C., Mat. Sci. For. 105-110 (1992) 1397.

[5] van Veen A., Schut H. , de Vries J., Hakvoort R.A. and IJpma M.R., Positron beams for solids and surfaces, AIP Conf. Proc. 218, London, Ontario, Canada 3-6 July 1990 (AIP, New York, 1990), pp. 171-196.

[6] van Veen A. and Schut H., "VEPFIT applied to positron depth profiling problems", this workshop.

[7] Microsoft Excel User's Guide for version 4.0, Book 1 and 2. 